\section{NGD-A FORTRAN IV program for fitting the normal generated distribution}

H. CHARLES ROMESBURG and KIM MARSHALL
Computer Center, Utah State University, Logan, Utah 84322

and

\section{JEROME V. DEGRAFF \\ U.S. Forest Service, Richfield, Utah 84701}

The distribution of a random variable $p$, where $p$ is constrained on the domain $0<p<1$, is often of interest. For example, $p$ could be a subjectively estimated probability of a subject's preference for an item, or it could be the fraction of questions that a subject answers correctly on a test. In examples like these, the beta distribution is often used for the probability density function (PDF) of $p$, that is, the distribution of the random variable p over a set of subjects. However, the two-parameter $\left(\xi, \tau^{2}\right)$ normal generated distribution's PDF (Chiu, 1974; Romesburg, 1976) follows the same family of curves that the beta distribution's PDF follows (Figure 1), and it has features that favor it in application: (1) Unbiased maximum likelihood estimates (MLEs) of the two normal generated distribution parameters are available, whereas MLEs of the two beta distribution parameters are biased and computationally hard to find (Gnanadesikan, Pinkham, \& Hughes, 1967). (2) The distributions of the MLE parameter estimates of $\xi$ and $\tau^{2}$ can be obtained and, moreover, are common ( $\xi$ is normally distributed, whereas $\hat{\tau}^{2}$ is, when scaled by a constant, chi-square distributed, allowing hypotheses tests of the equality of the parameters among two or more distributions). (3) The normal generated distribution cumulative distribution function (CDF) is readily found and evaluated using properties of the normal distribution, whereas the beta distribution's CDF requires special tables.
The PDF of the normal generated distribution, $q(p)$, is written as follows:

$q(p)=\phi\left\{\frac{1 / \tau\left[\Phi^{-1}(p)-\xi\right]}{\tau \phi\left[\Phi^{-1}(p)\right]}\right\} \quad \begin{aligned} & 0<p<1 \\ & -\infty<\xi<\infty \\ & 0<\tau^{2}<\infty,\end{aligned}$

where $\xi$ and $\tau^{2}$ are the parameters, and $\phi(\cdot)$ and $\Phi(\cdot)$ are the standard normal PDF and CDF, respectively. Defining $c=\xi /\left(1+\tau^{2}\right)^{1 / 2}$ and $w=\tau^{2} /\left(1+\tau^{2}\right)$, the mean, $E(p)$, and variance, $\operatorname{Var}(p)$, of $q(p)$ are

$$
\begin{gathered}
\mathrm{E}(\mathrm{p})=\Phi(\mathrm{p}) \\
\operatorname{Var}(\mathrm{p})=\operatorname{Pr}[\mathrm{y} \leqslant \mathrm{c}, \mathrm{z} \leqslant \mathrm{c} ; \mathrm{w}]-\mathrm{E}^{2}(\mathrm{P}),
\end{gathered}
$$

where $\mathrm{y}$ and $\mathrm{z}$ have a joint bivariate normal distribution with zero means, unit variances, and correlation $w$. $\operatorname{Pr}[\cdot]$ denotes the joint bivariate probability. For a set of $n$ sample proportions $p_{1}, p_{2}, \ldots, p_{n}$, the MLE parameter estimates are

$$
\hat{\xi}=\sum_{i=1}^{n} \Phi^{-1}\left(p_{i}\right) / n
$$

and

$$
\hat{\tau}^{2}=\sum_{\mathrm{i}=1}^{\mathrm{n}}\left[\Phi^{-1}\left(\mathrm{p}_{\mathrm{i}}\right)-\xi\right]^{2} /(\mathrm{n}-1) .
$$

The CDF, $Q(p)$, by direct integration of Equation 1, is

$$
\mathrm{Q}(\mathrm{p})=\Phi\left\{1 / \tau\left[\Phi^{-1}(\mathrm{p})-\xi\right]\right\} \text {. }
$$

The estimation of parameters by Equations 4 and 5 , the calculation of the mean and variance of $q(p)$ by Equations 2 and 3, and the evaluation of the PDF and CDF by Equations 1 and 6 depend only upon the sample proportions, the standard normal distribution PDF $\phi(\mathrm{p})$ and $\operatorname{CDF} \Phi(\mathrm{p})$, the inverse $\operatorname{CDF} \Phi^{-1}(\mathrm{p})$, and the joint bivariate normal probability $\operatorname{Pr}[\cdot]$. However, the repeti-
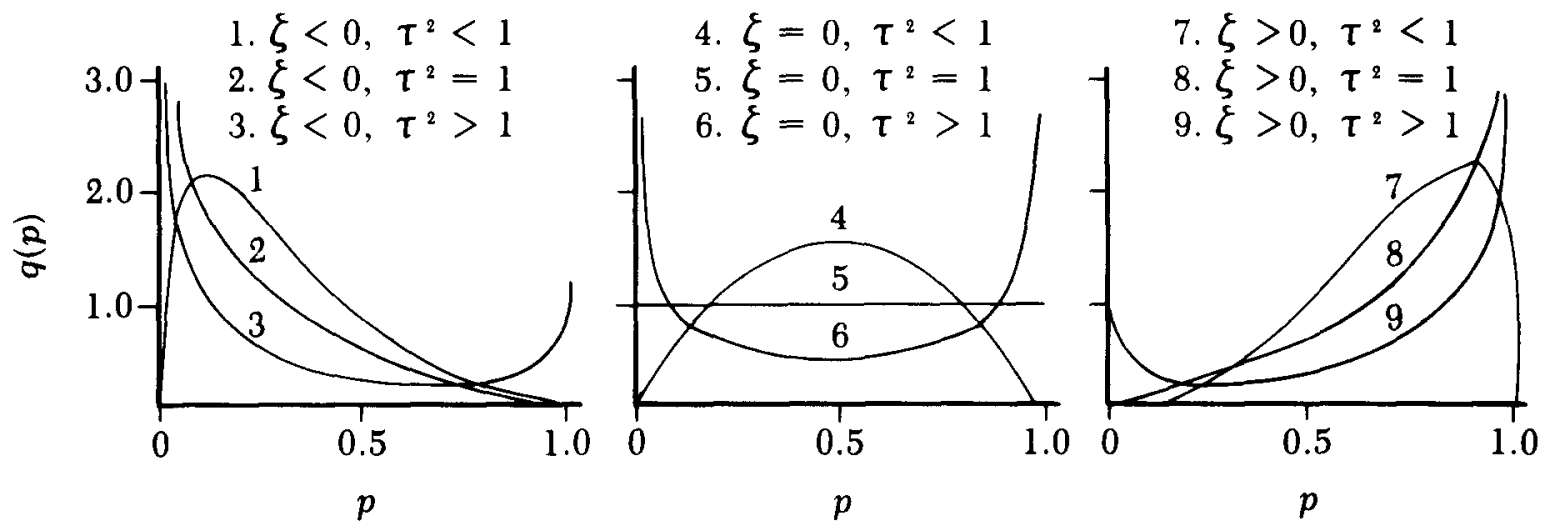

Figure 1. Typical curves for the normal generated distribution PDF $q(p)$ as a function of the parameter space: $\xi<,=,>0$; $\tau^{2}<,=,>1$. 
tive evaluation of these functions by hand, using interpolated tables is tedious and error prone. The computer program NGD was written for this reason.

Input. The program requires the sample proportions. Cases can be stacked.

Output. The program provides estimates $\xi$ and $\hat{\tau}^{2}$ and estimates $E(p)$ and $\operatorname{Var}(p)$. A table giving $q(p)$ and $Q(p)$ vs. $p$ is given, and a graphing routine plots $q(p)$ and $Q(p)$ vs. $p$ on a line printer.

Restrictions. The program is written in standard FORTRAN IV. Transfer to other computer systems should require minimal or no changes. The program is composed of 250 card images.

Availability. A copy of the listing, the input instructions, a tutorial that explains the NGD in more detail, and sample output containing test problems can be obtained free of charge by writing to $H$. Charles Romesburg, Computer Center, UMC 37, Utah State University, Logan, Utah 84322.

\section{REFERENCES}

Chiv, W. K. A new prior distribution for attributes sampling. Technometrics, 1974, 16, 93-102.

Gnanadesikan, R., Pinkham, R. S., \& Hughes, L. P. Maximum likelihood estimation of the parameters of the beta distribution from smallest order statistics. Technometrics, 1967, 9, 607-602.

RomesBurG, H. C. Use of the normal generated distribution for estimating population survival. Journal of Theoretical Biology, 1976, 61, 447-457.

(Accepted for publication April 10, 1980.) 